\title{
Simulation Model to Calculate Bird-Aircraft Collisions and Near Misses in the Airport Vicinity
}

\author{
Isabel C. Metz ${ }^{1,2, *}$, Thorsten Mühlhausen ${ }^{1}$, Joost Ellerbroek ${ }^{2}$, Dirk Kügler ${ }^{1}$, \\ Hans van Gasteren ${ }^{3} \mathbb{D}$, Jan Kraemer ${ }^{1}$ and Jacco M. Hoekstra ${ }^{2}$ \\ 1 Institute of Flight Guidance, German Aerospace Center (DLR), Lilienthalplatz 7, \\ 38108 Braunschweig, Germany; thorsten.muehlhausen@dlr.de (T.M.); dirk.kuegler@dlr.de (D.K.); \\ janosch.kraemer@gmail.com (J.K.) \\ 2 Control \& Simulation Department, Faculty of Aerospace Engineering, Delft University of Technology, \\ Kluyverweg 1, 2629 HS Delft, The Netherlands; j.ellerbroek@tudelft.nl (J.E.); j.m.hoekstra@tudelft.nl (J.M.H.) \\ 3 Royal Netherlands Air Force, P.O. Box 8762, 4820 BB Breda, The Netherlands; jr.v.gasteren@mindef.nl \\ * Correspondence: isabel.metz@dlr.de
}

Received: 23 September 2018; Accepted: 21 October 2018; Published: 25 October 2018

\begin{abstract}
Annually, thousands of birds collide with aircraft. The impact usually has lethal consequences for the bird, the involved aircraft can experience severe damage. The highest bird strike risk occurs at low altitudes. Therefore, aircraft within the airport area as well as the adjacent approach and departure corridors are especially vulnerable to collisions with birds. To analyse risk-reducing measures in these areas, a fast-time bird strike simulation environment was developed. An open-source Air Traffic Management simulator was enhanced with a model to represent bird movements and to recognize bird strikes. To confirm the reproducibility of the outcome, Monte Carlo simulations were performed. They included bird movement data from one year and air traffic flight plans for various air traffic volumes. The number of strikes and near misses showed an expected variance within the individual replications. The results indicate that the predictability of the number of strikes and near misses increases with rising number of birds, and rising air traffic intensity. Thus, by considering simulation scenarios including bird movement information from all seasons and a sufficient air traffic volume, the described set-up leads to stable results.
\end{abstract}

Keywords: aviation safety; bird movement model; bird strike; fast-time simulation; monte carlo simulation; near miss; risk calculation; runway capacity

\section{Introduction}

Collisions between birds and aircraft, so called bird strikes, pose a risk for both involved parties. For birds, the collision with an aircraft is a lethal danger [1]. Annually, thousands of birds fall victim to bird strikes (cf. e.g., [2]). The consequences for the involved aircraft are usually not as severe. Still, a bird strike can result in a total loss of an aircraft and therefore is life-threatening for humans as well $[2,3]$. Due to their impact on operations and their potential to cause severe damage, if not accidents [3], the aviation community strongly invests in measures to minimize the risk of bird strikes [4,5]. The majority of bird strikes occur at heights below $1000 \mathrm{~m}$ and therefore during departure or approach [5-7]. Hence, the focus of these measures mainly lies on the area within the airport fences. The International Civil Aviation Organization (ICAO) requires airports to maintain a wildlife strike prevention programme [8]. Research shows that the resulting mitigation measures have reduced the risk of bird strikes at airports [4]. However, in the area adjacent to the airport boundaries, where approaching and departing aircraft fly below a height of $1000 \mathrm{~m}$, the operational options to reduce the bird strike risk are limited [9]. In parts, this has contributed to an increasing trend in damaging strikes outside the airport boundaries over the past years $[2,4,9]$. Furthermore, the risk of damage rises with 
increasing kinetic energy. Due to larger bird sizes and higher velocities of aircraft at higher altitudes, the risk of a damaging strike is increasing as well [4]. To reduce the risk of bird strikes in the entire critical area below $1000 \mathrm{~m}$, this study aims at investigating the feasibility of involving Air Traffic Control (ATC) and pilots into the bird strike prevention process. For this purpose, a system of monitoring the aerial bird movements and providing real-time information about bird activity in the approach and departure corridors could be implemented. By applying an algorithm that uses birds' observed trajectories to predict their probable short-term future progression, risk levels based on the likelihood of a collision could be derived. In case of a high predicted risk level, for example, ATC could delay the take-off clearances. Alternatively, the risk information could be forwarded to pilots as an input for their decision-making. According to McKee et al. [5], ATC and pilots are reluctant of active involvement in operational bird strike prevention due to an anticipated increase in workload. Also airline and airport operators are hesitant. Among others, a decrease in runway throughput following the delaying of aircraft is anticipated [5]. In ongoing research, the Federal Aviation Administration (FAA) has addressed, among others, the potential rise workload for controllers when applying such a concept. Initial results from Human in the Loop (HIL) simulations indicate no increase in workload but rather potential workload savings [10]. The research presented here focuses on the effect on runway capacity. Furthermore, the potential increase in safety will be investigated. Thereby, the primary focus lies on an implementation where only departing aircraft are delayed. First, the consequences of a bird strike in this flight phase are most critical [11,12]. Second, delaying approaches is operationally inconvenient and does not necessarily improve safety. To delay an approaching aircraft would result in a go-around of that aircraft. If it collided with a bird during that manoeuvre, this could lead to more severe consequences than a strike during landing. This is, because an aircraft performing a go-around has to prolong its flight under increased thrust requirements. This could be critical in case of damage. On the other hand, a landing aircraft has the shortest path to the ground which reduces the time airborne after a potential strike [13].

The delaying of aircraft leads to a reduction of the possible throughput of a runway. For runways with a high traffic demand, this can result in a critical limitation of the runway capacity. Consequently, the operational feasibility of such a bird alert system depends on the balance between the potential of risk reduction and the decrease in runway throughput.

To evaluate the feasibility of such a system, a fast-time simulation environment representing air traffic and bird movements was developed in Metz et al. [14] and verified with an initial dataset for bird and air traffic movements. The tested data included aircraft movements at a single-runway airport with four different air traffic densities. Bird movement was obtained from weather and avian radar for one week per month within one year. To obtain results for a baseline scenario, aircraft and bird trajectories were deterministic, i.e., no uncertainties in movements were included. Up to the authors' knowledge, this is the first study evaluating the risk on bird strikes based on simulations of bird movements and air traffic. The results of that study are promising; however, the performed verification considered a limited dataset only. As bird strikes are relatively rare events, their occurrence is, in parts, subject to chance. Therefore, there is a risk that the outcome of the initial simulations in Metz et al. [14] is sensitive to the specific constellation of the input data, and thus is not representative.

The objective of this study was to confirm the reproducibility of the simulation results and to establish a convincing conclusion about the robustness of the developed set-up. For this purpose, Monte Carlo simulations were performed. Furthermore, the focus of the analysis was extended to consider near misses-dangerously close vicinity between birds and aircraft-as well. This increases the number of occurrences and thus the dataset available for statistical analysis considerably, as near misses take place much more often than actual collisions. Bird movement information from one year was randomized and combined with four air traffic volumes. It was hypothesized that due to the random nature of bird strike occurrences, variations in bird strike numbers would occur within the individual replications of the simulation. 


\section{Materials and Methods}

\subsection{Simulation Environment}

To simulate the risk of bird strikes, a simulation environment including the movements of both, birds and air traffic, was developed in Metz et al. [14]. The environment's current set-up represents a situation around an airport where no bird alert system is active. Consequently, it can serve as a baseline for studies involving such a system or other risk-reducing measures relying on bird movement information. A summary of the simulation environment is presented in the following section.

\subsubsection{Simulation Platform}

For this study, the BlueSky Open Air Traffic Simulator, a development by Delft University of Technology, was used. This simulator facilitates the analysis and visualization of air traffic flows as well as Air Traffic Management (ATM) concepts. Air traffic can be simulated in real- and fast-time, including validated aircraft performance characteristics, all based on open sources and open data $[15,16]$. Hence, the representation of air traffic required for this study is already provided by the simulation platform. In Metz et al. [14], BlueSky was enhanced to represent bird movements and to record collisions between birds and aircraft.

Similar to the existing aircraft representation, birds are modelled as point-masses within the simulation. No bird behaviour or reaction to aircraft is modelled. This simplification is expected to lead to an overestimation of bird strikes in the simulation. Data from different studies suggest that in reality, some bird species show avoidance reactions to approaching aircraft and sometimes manage timely escapes [17-19].

To detect collisions between birds and aircraft, protected zones around every aircraft and every bird are defined. Whenever an aircraft and a bird penetrate each other's protected zone, a bird strike is recorded and the respective bird removed from the simulation, as it is assumed that the collision is lethal for the bird. Additionally, so called near misses are counted. A near miss, as defined by the Royal Netherlands Air Force and used within this study, occurs when the separation between an aircraft and a bird becomes smaller than $50 \mathrm{~m}$. Near misses happen more often than collisions. Hence, by taking the former into account, a larger dataset is available for analysis. However, due to missing reports about near misses, no comparison between simulated and real data could be performed.

\subsubsection{Bird Movement}

To represent bird movements within the simulation, data from two different sources is used to cover the extended airport area up to a height of $1000 \mathrm{~m}$. This is the area where the risk of bird strike is highest [5,6]. From ground level to a height of $200 \mathrm{~m}$, an avian radar located at an airport serves as input. Avian radar is specifically designed to track birds up to distances of $11 \mathrm{~km}$ and heights of $1.5 \mathrm{~km}$ [20]. However, due to radar shadowing and limitations in tracking single birds, especially close to the ground and with increasing distance from the radar [21,22], not all birds present are actually represented in the avian radar data. The reduced number of individual birds close to the ground is not addressed in this study. Therefore, the actual number of birds and the related collision risk is expected to be slightly underestimated on the airport grounds. To receive more representative data for birds at larger distances, i.e., outside the airport boundaries, the avian radar data was complemented with information from weather radar. Weather radar has been used for the quantification of bird movements for several decades $[23,24]$ as well as for studies on large-scale bird migration (cf. e.g., [25,26]). Aircraft fly higher with increasing distance from the airport and thus the radar location. In correspondence to the presentation of the weather radar data, which is provided in altitude intervals of $200 \mathrm{~m}$, weather radar data is used to represent bird movement from $200 \mathrm{~m}$ to $1000 \mathrm{~m}$. This study used data from two Dutch radars, namely the avian radar at Eindhoven airport and the weather radar located in De Bilt. The output from the avian radar provides two-dimensional tracks for individual birds and flocks. To generate three-dimensional movements, height information 
was added based on distributions observed in Shamoun-Baranes et al. [7]. The weather radar output contains information about bird density as well as average directions and velocities of birds. To obtain bird movement information from this radar, the data was processed based on the methods developed in van Gasteren et al. [27] and Dokter et al. [28].

\subsubsection{Flock Composition}

Many bird species move in flocks, especially during daytime [29,30]. The weather radar data contains information about the total number of birds present in the airspace. However, information about the local distribution and size of the birds is not detectable by the system. The avian radar data distinguishes between individuals and flocks. However, the generated output neither contains the number of birds within a flock nor the size of these birds. Furthermore, neither of the radars identifies species. As the size of birds and flocks is strongly relevant to determine potential damage resulting from bird strikes [31], a model to estimate flock sizes was developed [14]. This model is based on a study on bird migration over the Netherlands between 1981 and 1983 [32]. The study is the most complete source offering information on flock compositions of birds in the Netherlands. Furthermore, it considers bird movements below and above $200 \mathrm{~m}$ separately. This perfectly fits to the boundaries between avian and weather radar selected for this study. Although the distribution between individual species might have changed in the time since the study, it is still a valuable source for this research.

To retrieve flock information for the birds flying in the range of the avian radar, the top 15 species flying below $200 \mathrm{~m}$ were selected, representing $89 \%$ of all birds in this category [32]. Of the 51 species observed by Lensink and Kwak [32], 16 were shown to fly above $200 \mathrm{~m}$. For 14 of them, corresponding to $97 \%$ of all individuals, flock size information was available. Based on their average weight obtained from Dunning [33], all chosen species were assigned either to the size class small (up to $0.085 \mathrm{~kg}$ ), medium $(0.085 \mathrm{~kg}-1.150 \mathrm{~kg}$ ) or large (above $1.150 \mathrm{~kg}$ ) as defined by the aviation authorities (FAA, 2011). All species considered are presented in Table 1.

For every species covered by the report, the share of birds flying individually respective within flocks is provided. Furthermore, average flock sizes per species are contained. To establish the relative abundance of individual flying birds, the percentages of individual flying bird per species were summed by extrapolation from the proportion of each species in the population. The same was applied for birds flying in flocks.

To determine the number of birds within a flock, the species considered for this study were sorted by average flock size and categorized into three groups with similar flock sizes. Subsequently, the average flock size within every of these groups was calculated. Therefore, the flock sizes of every species within a group was weighted with the species' share within its group. Subsequently, the distribution between small, medium and large birds per flock size group was determined. For this purpose, the shares of species within each size category was summed up. The result corresponds to the share of the respective size category within the flock size group.

The same procedure was followed for flocks in the range of the avian radar. The size distribution of birds flying individually in the range of the weather radar was calculated correspondingly by including all birds considered (cf. Table 1). 
Table 1. Bird species chosen for modelling flock compositions. Source: [32].

\begin{tabular}{|c|c|c|c|c|c|c|c|}
\hline \multicolumn{4}{|c|}{ Range Avian Radar (0 m-200 m) } & \multicolumn{4}{|c|}{ Range Weather Radar (200 m-400 m) } \\
\hline Species & Ratio $^{a}$ & $\begin{array}{c}\text { Cumulated } \\
\text { Ratio }\end{array}$ & Bird Size & Species & Ratio $^{b}$ & $\begin{array}{c}\text { Cumulated } \\
\text { Ratio }\end{array}$ & Bird Size \\
\hline $\begin{array}{l}\text { common } \\
\text { chaffinch }\end{array}$ & 25.94 & 25.94 & small & redwing & 25.40 & 25.40 & small \\
\hline $\begin{array}{l}\text { common } \\
\text { starling }\end{array}$ & 11.49 & 37.43 & small & lapwing & 23.58 & 48.98 & medium \\
\hline meadow pipit & 8.40 & 45.83 & small & $\begin{array}{l}\text { black-headed } \\
\text { gull }\end{array}$ & 16.41 & 65.39 & medium \\
\hline redwing & 7.42 & 53.25 & small & rook & 12.67 & 78.06 & medium \\
\hline barn swallow & 4.68 & 57.93 & small & $\begin{array}{l}\text { eurasian } \\
\text { skylark }\end{array}$ & 4.02 & 82.08 & small \\
\hline $\begin{array}{l}\text { common } \\
\text { house martin }\end{array}$ & 4.56 & 62.49 & small & $\begin{array}{l}\text { western } \\
\text { jackdaw }\end{array}$ & 3.31 & 85.39 & medium \\
\hline $\begin{array}{l}\text { black-headed } \\
\text { gull }\end{array}$ & 4.19 & 66.68 & medium & $\begin{array}{l}\text { common } \\
\text { wood pigeon }\end{array}$ & 3.18 & 88.57 & medium \\
\hline $\begin{array}{l}\text { common } \\
\text { wood pigeon }\end{array}$ & 4.07 & 70.75 & medium & $\begin{array}{l}\text { common } \\
\text { chaffinch }\end{array}$ & 3.05 & 91.62 & small \\
\hline $\begin{array}{l}\text { eurasian } \\
\text { skylark }\end{array}$ & 3.98 & 74.73 & small & common swift & 2.68 & 94.30 & small \\
\hline lapwing & 3.91 & 78.64 & medium & fieldfare & 1.78 & 96.08 & medium \\
\hline red crossbill & 2.81 & 81.45 & medium & $\begin{array}{l}\text { european } \\
\text { herring gull }\end{array}$ & 1.19 & 97.27 & large \\
\hline $\begin{array}{l}\text { common } \\
\text { linnet }\end{array}$ & 2.52 & 83.97 & small & buzzard & 0.93 & 98.20 & large \\
\hline fieldfare & 2.47 & 86.44 & medium & $\begin{array}{l}\text { eurasian } \\
\text { sparrowhawk }\end{array}$ & 0.92 & 99.12 & medium \\
\hline common swift & 1.71 & 88.15 & small & $\begin{array}{l}\text { common } \\
\text { house martin }\end{array}$ & 0.88 & 100.00 & small \\
\hline $\begin{array}{l}\text { eurasian } \\
\text { siskin }\end{array}$ & 1.33 & 89.48 & small & & & & \\
\hline
\end{tabular}

These described distributions for flock and bird sizes are valid for daytime. During the night, only a few birds fly in flocks. Most birds travel alone or with large distances between the individuals [29,30,34]. The study of Lensink and Kwak [32] does not cover nocturnal migration. Hence, to represent bird distribution during the night, information was obtained from Hüppop et al. [35]. This paper describes a distribution between birds flying individually and in flocks during the night. Furthermore, for the birds that do fly in flocks during the night, three ranges of expected flock sizes for nocturnal migration are presented. These were used to calculate mean flock sizes for this study. Bird sizes per flock type were calculated based on the number of all birds considered in this study (cf. Table 1). 
During the generation of the bird movement information prior to the simulation, the distributions for diurnal and nocturnal flock sizes were applied depending on civil twilight. Tables 2 and 3 summarize the flock size distributions for the avian radar, Tables 4 and 5 for the weather radar. Figure 1 visualizes the resulting output after the processing of the avian and weather radar data.

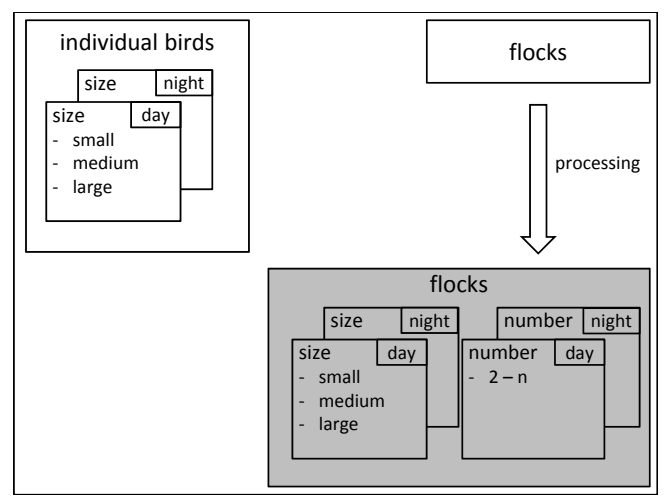

(a) Avian radar

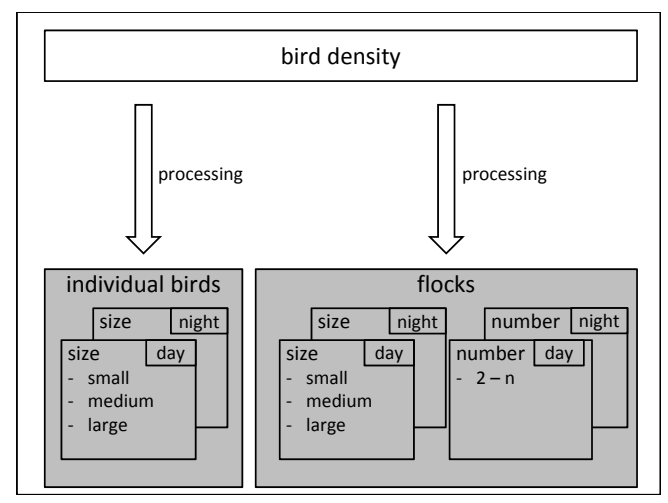

(b) Weather radar

Figure 1. Steps for retrieving flock size distributions (white: input information. grey: processed information). Avian radar (a): Input contains distribution between individual birds and flocks. For individual birds, size information for day and night is contained. Numbers of birds per flock as well as body size distribution within flocks were modelled. Weather radar (b): Input contains bird density. Distribution between birds flying individually and in flocks were modelled for day and night as well as are represented bird sizes and number of birds within flocks.

Table 2. Determined flock size groups within the range of the avian radar (0-200 $\mathrm{m})$ during daytime.

\begin{tabular}{lccccc}
\hline Group Identifier & $\begin{array}{c}\text { Represented } \\
\text { Birds (\%) }\end{array}$ & $\begin{array}{c}\text { Average } \\
\text { Flock Size (-) }\end{array}$ & $\begin{array}{c}\text { Small Birds } \\
\mathbf{( \% )}\end{array}$ & $\begin{array}{c}\text { Medium Birds } \\
\mathbf{( \% )}\end{array}$ & $\begin{array}{c}\text { Large } \\
\text { Birds (\%) }\end{array}$ \\
\hline I & 48 & 3.61 & 10 & 90 & 0 \\
II & 25 & 6.55 & 57 & 43 & 0 \\
III & 27 & 10.86 & 83 & 17 & 0 \\
\hline
\end{tabular}

Table 3. Determined flock size groups within the range of the avian radar (0-200 m) during night time.

\begin{tabular}{|c|c|c|c|c|c|}
\hline Group Identifier & $\begin{array}{l}\text { Represented } \\
\text { Birds }^{\text {a }}(\%)\end{array}$ & $\begin{array}{c}\text { Average } \\
\text { Flock Size }^{\text {b }}(-)\end{array}$ & $\underset{(\%)}{\text { Small Birds }}{ }^{c}$ & 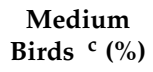 & $\begin{array}{c}\text { Large Birds } \\
(\%)\end{array}$ \\
\hline I & 51 & 15.00 & 80 & 20 & 0 \\
\hline II & 26 & 7.50 & 80 & 20 & 0 \\
\hline III & 23 & 3.50 & 80 & 20 & 0 \\
\hline
\end{tabular}

${ }^{a}$ Number of birds flying in respective groups. Source: Hüppop, et al. (2006); ${ }^{b}$ source: Hüppop, et al. (2006);

${ }^{\mathrm{c}}$ source: Lensink and Kwak (1985).

Table 4. Determined flock size groups within the range of the weather radar (200 m-1000 m) during daytime. Average flock sizes for groups I-III were calculated by including all birds in the respective species. To separate individually flying birds, they were subtracted from the birds represented in group I-III and categorized into group IV.

\begin{tabular}{cccccc}
\hline Group Identifier & Represented Birds (\%) & Average Flock Size (-) & Small Birds (\%) & Medium Birds (\%) & Large Birds (\%) \\
\hline I & 21 & 1.87 & 24 & 34 & 42 \\
II & 22 & 6.49 & 48 & 52 & 0 \\
III & 51 & 13.51 & 34 & 66 & 62 \\
IV & 6 & 1.00 & 36 & 2 \\
\hline
\end{tabular}


Table 5. Determined flock size groups within the range of the weather radar (200 m-1000 m) during night time. Group IV represents individually flying birds.

\begin{tabular}{|c|c|c|c|c|c|}
\hline Group Identifier & Represented Birds a (\%) & Average Flock Size ${ }^{\text {b }}(-)$ & Small Birds ${ }^{c}(\%)$ & Medium Birds $^{c}$ (\%) & Large Birds ${ }^{c}(\%)$ \\
\hline I & 10 & 3.50 & 27 & 37 & 36 \\
\hline II & 5 & 7.50 & 27 & 37 & 36 \\
\hline III & 5 & 15.00 & 27 & 37 & 36 \\
\hline IV & 80 & 1.00 & 27 & 37 & 36 \\
\hline
\end{tabular}

${ }^{a}$ number of birds flying in respective groups. Source: Hüppop, et al. (2006); ${ }^{\text {b }}$ source: Hüppop, et al. (2006);

c source: Lensink and Kwak (1985).

\subsection{Monte Carlo Simulations}

Bird strikes are relatively rare events. Depending on the ecosystems surrounding an airport, bird strike rates expressed in number of bird strikes per 10,000 aircraft movements generally vary between two and 20 (cf. e.g., [2,36]). Hence, the occurrence of a bird strike is, in parts, subject to chance. Consequently, to obtain a convincing conclusion about the robustness of a system simulating bird strikes, a large sample size is required. However, only a limited dataset was available for this study. Therefore, the stability of the model was evaluated by performing Monte Carlo simulations. These included various bird movement and air traffic information. The risk of bird strikes strongly depends on the season [5,37]. For this reason, bird movement information was generated for one day per month from October 2015 to September 2016. Per month, the day with the average number of birds per month was chosen. Another risk-contributing factor to bird strike occurrences is the number of aircraft in the airspace [38]. Therefore, flight plans representing different daily traffic volumes on a single-runway airport, were generated. For this purpose, real flight plans from four airports were obtained from the Demand Data Repository (DDR) 2 data base of the European Organization for the Safety of Air Navigation (EUROCONTROL), representing the air traffic volumes high (954 movements), medium (501 movements), low (305 movements) and very low (79 movements) within the simulation (cf. [14]). The flight plans were mapped to Eindhoven airport, another single-runway airport, where the bird movement information originates from. Within all simulations, the runway was operated in its northern direction 03 , where 03 refers to the runway heading of 30 degrees. The simulated area includes the approach corridors of runway 03, beginning at the Initial Approach Fixes (IAFs). For departing aircraft, the Standard Instrument Departures (SID) routes are included. Approaching air traffic is simulated from their Initial Approach Fix (IAF) until landing. Departing traffic is simulated from take-off until reaching one kilometer height. Birds from the weather radar are initialized and simulated over the entire area and removed as soon as they reach one of the area's boundary. The initialization and simulation of avian radar birds is determined by the input data. Collisions and near misses are counted within the entire area. The simulated area is visualized in Figure 2.

For all air traffic scenarios, identical airport opening hours were applied. By merging the 12 bird movement plans with the four traffic volumes, 48 scenarios resulted. This study aims at validating a set-up to evaluate the number of bird strikes actually happening at an airport. Therefore, trajectories of bird and aircraft are considered to be deterministic, i.e., no uncertainties regarding the actual positions are involved.

Within the Monte Carlo simulations, every scenario was replicated 100 times. All randomized parameters concentrated on the bird movement information. To maximize the randomness, all parameters which were not fixed by the input data, were randomized for the replications. Considering the birds from the avian radar, where a two-dimensional track is given, the initialized height was randomized. Regarding the birds from the weather radar, where only the number of birds is contained in the input data, the simulation's initial parameters for height, latitude, longitude, heading and speed were randomized. All randomizations were performed by applying uniform distributions within the ranges defined by Metz et al. [14] as summarized in Table 6. The uniform distribution was chosen to ensure an even distribution of the bird positions in all degrees of freedom. The resulting set-up for the Monte Carlo simulations is visualized in Figure 3. 
Table 6. Parameters randomized for the Monte Carlo replications and the ranges within which the randomizations were carried out. AR: avian radar; WR: weather radar. Source: [14].

\begin{tabular}{ccc}
\hline Parameter & Radar Source & Range \\
\hline altitude & AR, WR & respective altitude band \\
latitude & WR & entire area \\
longitude & WR & entire area \\
speed & WR & $\pm 6 \frac{m}{s}$ \\
heading & WR & $\pm 45^{\circ}$ \\
\hline
\end{tabular}

For consistency and reproducibility, all 100 iterations of the 48 scenarios took place with identical randomization seeds. The total number of simulation runs amounted to 4800 . To determine whether this number of replications was sufficient, the convergence of average bird strike and near miss numbers was assessed. Furthermore, the corresponding standard errors were evaluated. It was determined that the number of replications was sufficient, if a standard error below $5 \%$ would be reached. Figure 4 visualizes the results. Except for the very low scenario considering collisions, all scenarios reached the criterion of a standard error below $5 \%$ within the 100 replications. The very low scenario reached a standard error of $6 \%$ from replication 90 in relation to collisions. As this scenario is limited by a small sample size due to the small number of aircraft, the reduction in standard error for this scenario was defined as acceptable as well.

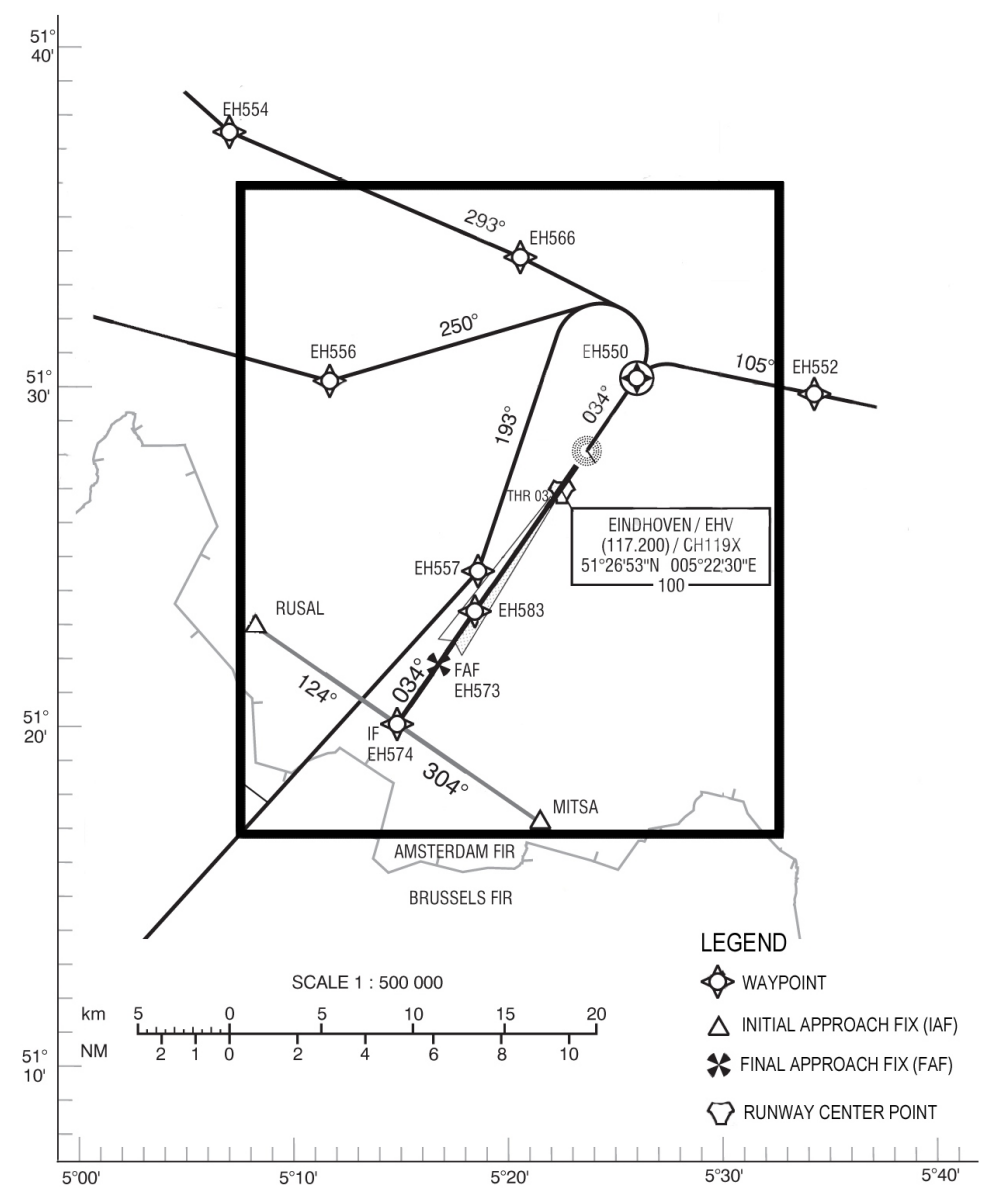

Figure 2. Area simulated for this study (own figure, based on [39]). 


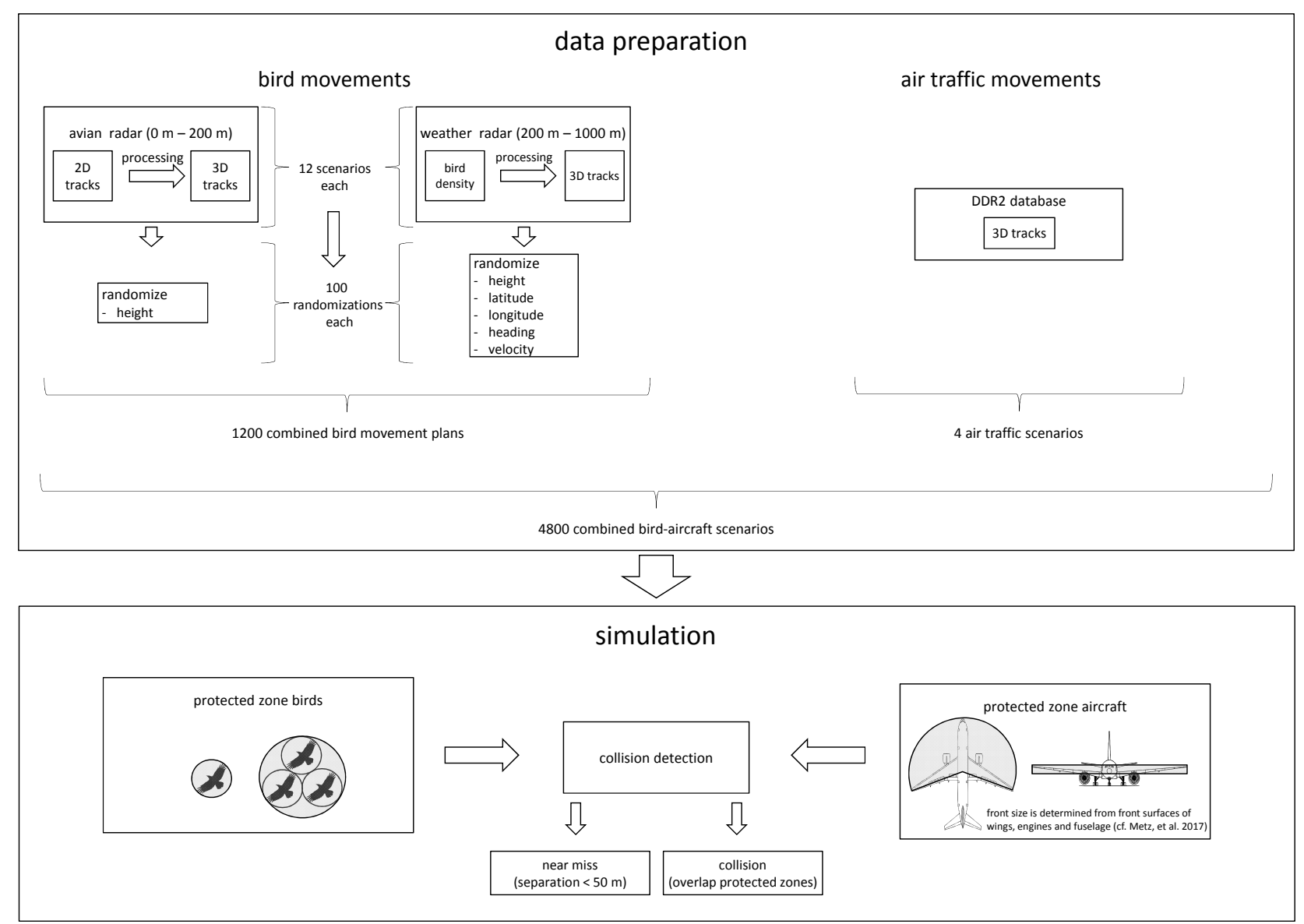

Figure 3. Method overview. Within the data preparation of bird movements, 12 scenarios of bird tracks are generated from weather and avian radar. By randomizing the input parameters 100 times, 1200 bird movement plans result. Air traffic trajectories from four single-runway airports are retrieved from EUROCONTROL's DDR2 and processed to air traffic scenarios. By combining the bird movement plans with the air traffic scenarios, 4800 bird-aircraft scenarios result. These serve as input for the fast-time simulation. Based on the definition of protected zones (cf. [14]), bird strikes and near misses are counted and recorded. 


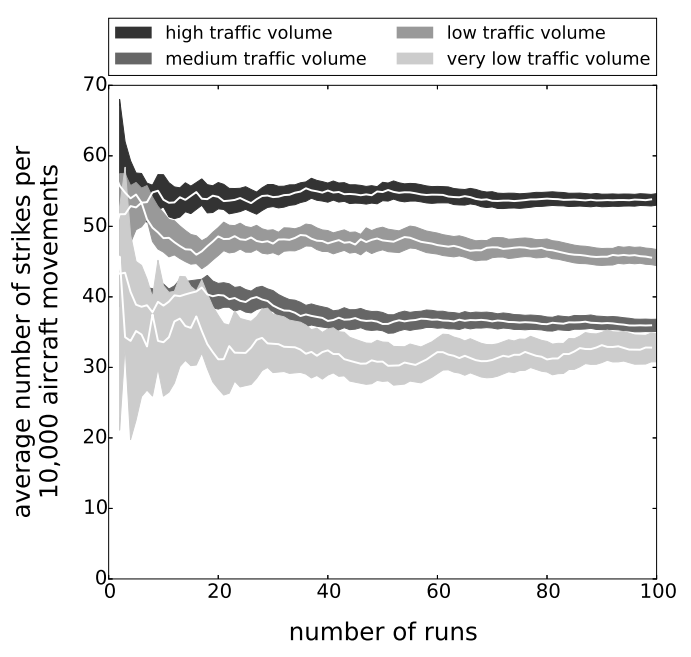

(a)

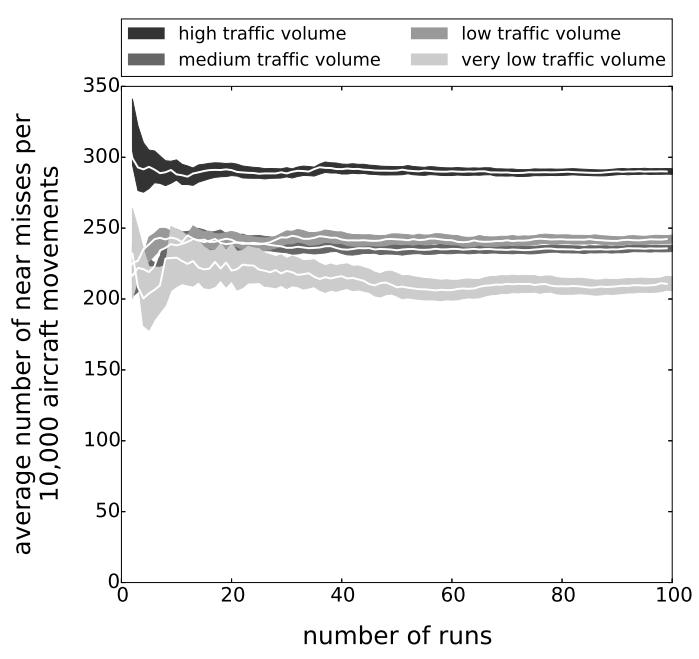

(b)

Figure 4. Convergence of average number of bird strikes and near misses over all simulation runs. White lines represent the average numbers, colored areas the standard error. (a) Bird strikes; (b) Near misses.

\section{Results}

To evaluate the bird strike risk within the simulation environment, the bird strike rate as well as the near miss rate were calculated for all scenarios. Thereby, all collisions and near misses within the defined area around the airport and below $1000 \mathrm{~m}$ (cf. Figure 2) were considered.

The average bird strike rate within the simulation lies between 30 and 40 strikes per 10,000 aircraft movements for the different traffic intensities. The average near miss rate lies between 190 and 220 near misses per 10,000 aircraft movements. Figure 5 summarizes these results. Regarding both, strikes and near misses, simulations including larger air traffic volumes have smaller ranges. The ranges for the near misses are higher than those for actual bird strikes. The ranges for near misses are higher than those for actual bird strikes.

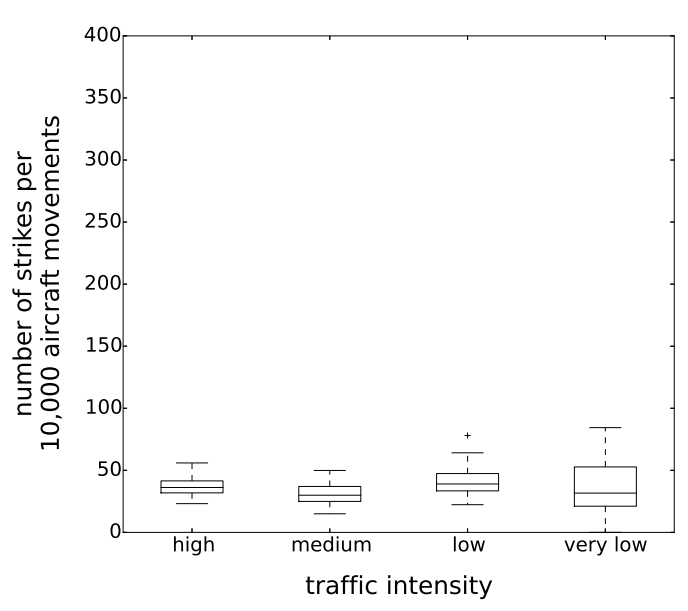

(a)

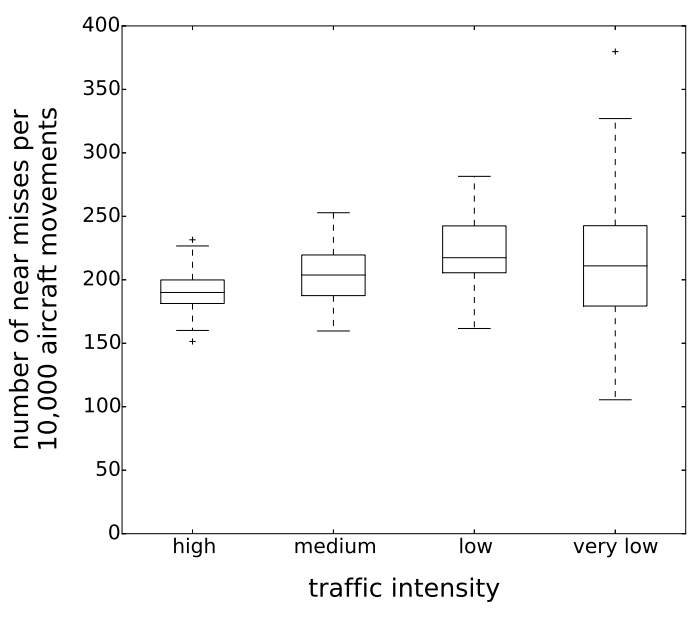

(b)

Figure 5. Boxplot values for the number of bird strikes over all Monte Carlo replications and for all traffic volumes. (a) Collisions; (b) Near misses.

The comparison of the simulation results to real data from Eindhoven airport reveals an overestimate of number of bird strikes within the simulation. Between 2007 and 2016, the average 
bird strike rate amounted to 12.33 for Eindhoven. This ratio includes local bird strikes reported to have occurred at the airport respective in its vicinity [40]. The simulation results of 30 to 40 strikes per 10,000 flights therefore correspond to an overestimate by factor 2.5 to 3.3. Due to missing reporting of near miss occurrences, no comparison between simulated and real data could be performed here.

Figure 6 provides an overview of the monthly strike and near miss rates per air traffic intensity. Seasonal variance is more visible for scenarios including larger air traffic volumes. Peaks for bird strikes occur in the migratory months March, April and October. The highest average number of collisions and near misses as well as the largest range occurs in June. As for the annual overview (cf. Figure 5), the ranges decrease with rising air traffic volumes.

To determine the dependency between number of birds and number of collisions/near misses, the respective Spearman correlations were calculated. There is an obvious deviation between number of birds and number of events in June (cf. [14], pp. 6-7). Therefore, the correlations were calculated once for the entire year and once after excluding the June data. Table 7 summarizes the correlations and their levels of significance for the resulting boxplot values. The correlations and their significance increase with higher air traffic volumes. Furthermore, higher values result regarding the near misses than regarding collisions.

Within the very low scenarios, no bird strike event took place in 7 iterations considering the entire year and in 13 iterations when excluding June. To obtain a valid correlation value, these iterations were removed for the calculations of the correlations between bird strikes and number of birds.

Table 7. Boxplot values regarding the correlations between number of birds and number of collisions/near misses for the different traffic volumes $\left(Q_{1}-Q_{3}\right.$ : boxplot quartiles; $r_{s}$ : Spearman correlation, $p$ : one-tailed $p$-value).

\begin{tabular}{|c|c|c|c|c|c|c|c|}
\hline & \multicolumn{2}{|c|}{$Q_{1}$} & \multicolumn{2}{|c|}{$Q_{2}$} & \multicolumn{2}{|c|}{$Q_{3}$} & \multirow{2}{*}{$\mathbf{n}$} \\
\hline & $r_{s}$ & $p$ & $r_{s}$ & $p$ & $r_{s}$ & $p$ & \\
\hline \multicolumn{8}{|c|}{ collisions, entire year } \\
\hline very low & -0.14 & 0.091 & 0.13 & 0.108 & 0.31 & 0.001 & 93 \\
\hline low & 0.07 & 0.251 & 0.21 & 0.017 & 0.38 & $<0.001$ & 100 \\
\hline medium & 0.23 & 0.011 & 0.36 & $<0.001$ & 0.51 & $<0.001$ & 100 \\
\hline high & 0.06 & 0.226 & 0.28 & 0.002 & 0.42 & $<0.001$ & 100 \\
\hline \multicolumn{8}{|c|}{ collisions, w/o June } \\
\hline very low & 0.00 & 0.5 & 0.19 & 0.036 & 0.40 & $<0.001$ & 87 \\
\hline low & 0.12 & 0.127 & 0.31 & 0.001 & 0.48 & $<0.001$ & 100 \\
\hline medium & 0.23 & 0.010 & 0.39 & $<0.001$ & 0.51 & $<0.001$ & 100 \\
\hline high & 0.23 & 0.01 & 0.43 & $<0.001$ & 0.59 & $<0.001$ & 100 \\
\hline \multicolumn{8}{|c|}{ near misses, entire year } \\
\hline very low & 0.16 & 0.055 & 0.30 & 0.001 & 0.49 & $<0.001$ & 100 \\
\hline low & 0.48 & $<0.001$ & 0.53 & $<0.001$ & 0.61 & $<0.001$ & 100 \\
\hline medium & 0.63 & $<0.001$ & 0.70 & $<0.001$ & 0.77 & $<0.001$ & 100 \\
\hline high & 0.43 & $<0.001$ & 0.52 & $<0.001$ & 0.58 & $<0.001$ & 100 \\
\hline \multicolumn{8}{|c|}{ near misses, w/o June } \\
\hline very low & 0.25 & 0.006 & 0.42 & $<0.001$ & 0.61 & $<0.001$ & 100 \\
\hline low & 0.59 & $<0.001$ & 0.66 & $<0.001$ & 0.75 & $<0.001$ & 100 \\
\hline medium & 0.71 & $<0.001$ & 0.78 & $<0.001$ & 0.85 & $<0.001$ & 100 \\
\hline high & 0.61 & $<0.001$ & 0.72 & $<0.001$ & 0.81 & $<0.001$ & 100 \\
\hline
\end{tabular}



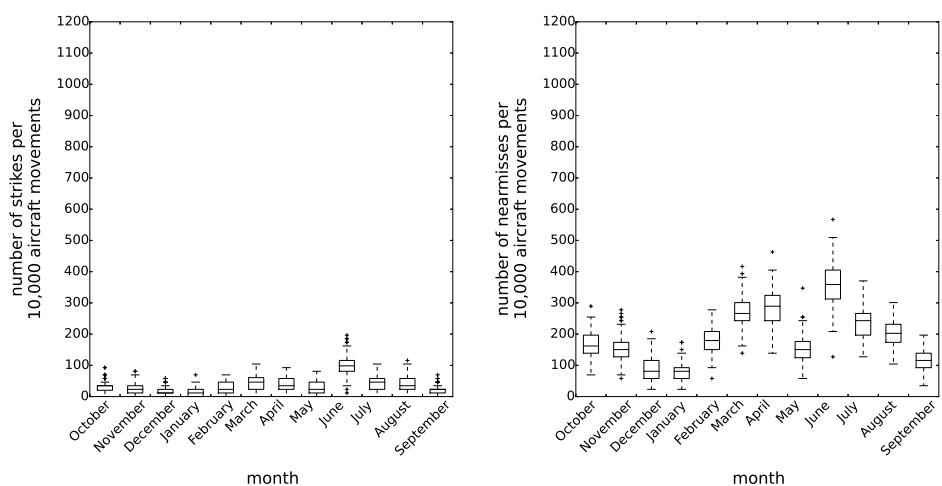

(a)
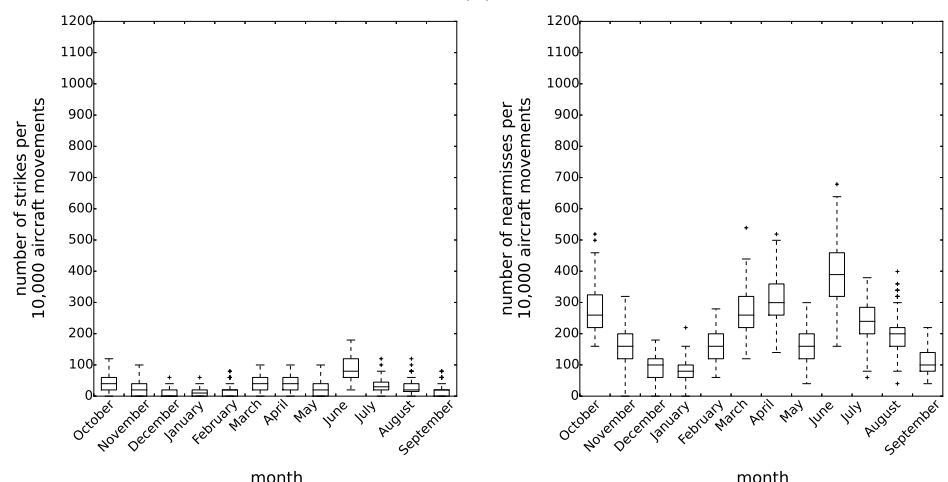

(b)
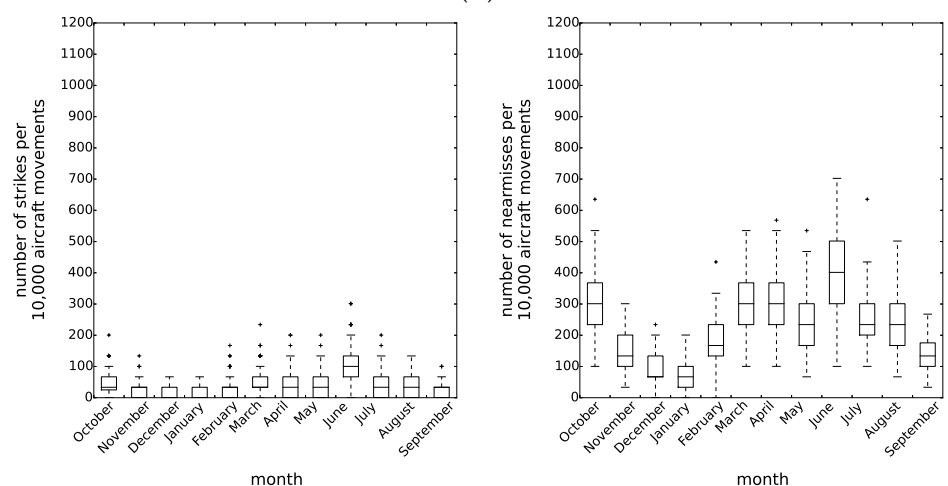

(c)
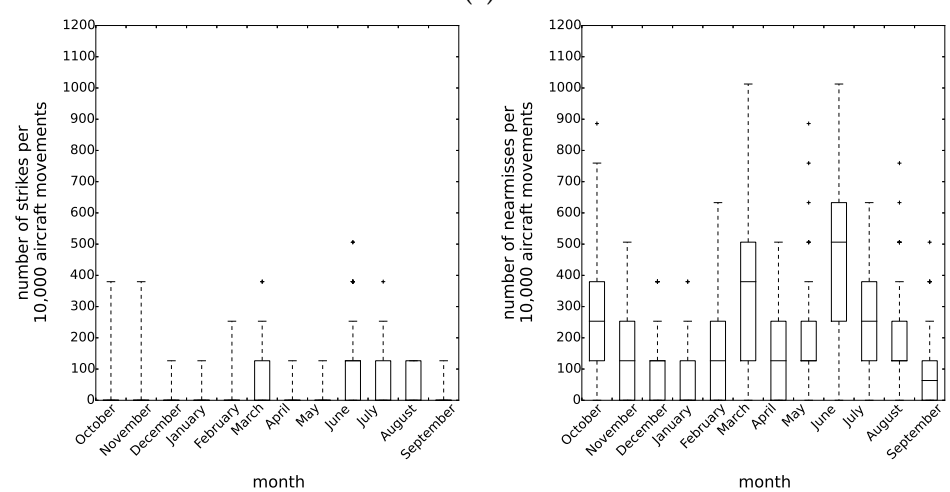

(d)

Figure 6. Boxplot values per month for the number of bird strikes over all Monte Carlo replications and for all traffic volumes. (a) Traffic scenario with high traffic density; (b) Traffic scenario with medium traffic density; (c) Traffic scenario with low traffic density; (d) Traffic scenario with very low traffic density. 


\section{Discussion}

In this study, Monte Carlo simulations were performed to evaluate the robustness of a simulation environment to simulate bird strikes and near misses as well as the reproducibility of the generated output. The simulations included bird movement data from one year and air traffic data representing airports with four different traffic volumes. In total, 48 scenarios with 100 replications each were performed. A test of the convergence of number of collisions and near misses as well as standard error confirmed that this number was sufficient.

The results of the Monte Carlo simulations were analysed with respect to the variance of bird strikes and near miss rates over the entire year as well as depending on the season. Furthermore, the correlation between the number of birds present in the airspace and the number of bird strikes and near misses was evaluated. As expected, it was found that there was variance in the bird strike rates among the individual simulation runs. The ranges decreased and the correlations increased with rising number of collisions and near misses for bird strikes and near misses with the latter producing more robust results. However, when comparing the number of bird strikes and near misses for the same traffic intensities in Figure 5, the ranges regarding the near misses are higher. This is related to the higher number of near misses as well as a higher range of the number of near misses. These results are reflected in the seasonal overview in Figure 6. The ranges decreased with higher traffic volume for both, bird strike and near miss rate. Regarding the same traffic volumes, the ranges were higher for near misses than for bird strikes.

The analysis of the Spearman correlation between number of birds and number of collisions/near misses revealed that, except for June, there is a coherence between these parameters. In June, where a high number of collisions and near misses take place in the simulation, a relatively low number of birds is active in the entire airspace. This was confirmed by an in-depth analysis of the data which revealed that the majority of bird strikes in June occur below a height of $200 \mathrm{~m}$, between $66 \%$ and $85 \%$ of all bird strikes (high: $81 \%$, medium: $78 \%$, low: $66 \%$, very low: $85 \%$ ). This is the area which is covered by the avian radar (cf. Section 2.1.2). Of all strikes below $200 \mathrm{~m}$ throughout the year, between $58 \%$ and $78 \%$ (high: $65 \%$, medium: $58 \%$, low: $66 \%$, very low: $78 \%$ ) take place in June. This is only partly reflected by an increase of bird movements below $200 \mathrm{~m}$. The number of birds present in the lowest altitude band lies only slightly above the average number of birds present per year (approximately $10 \%$ of all bird movements in the different scenarios). Hence, the reason for this significant increase in bird strikes is related to the geographical distribution of tracks rather than to the number of birds. This corresponds to international bird strike data which indicates that the fledging of young birds, which takes place in summer, leads to the highest risk of bird strikes. In the Netherlands, where the bird data originates from, most birds fledge in June [27,41,42]. Another contributing factor could be a contamination of the avian radar data by insect echoes. However, as no similar effect was observed for the other summer months, this seems unlikely. When excluding June for the analysis, the correlations between bird strikes and number of birds increase and become significant. With regard to near misses, where a higher number of events is available for analysis, except for the very low scenario, the average correlations between bird density and number of events are remarkably high. With the exception of June, this strengthens the argument for a higher risk of bird strikes with rising bird numbers throughout the year.

The average bird strike rates obtained in this study are higher than the ones in the initial analysis performed by Metz et al [14]. This deviation lies in an adaptation of the bird model that was performed for the Monte Carlo simulations to achieve a more realistic bird representation. By rerunning the initial simulations from [14] after including this adaptation, the bird strike rates lie in the same range as for the Monte Carlo simulations described in this paper.

The large ranges of the number of collisions and near misses as well as for the correlation between number of birds and number of collisions and near misses for the very low traffic volume indicate that the number of collisions and near misses is too low to gain representative and reproducible results in this scenario. Hence, for further studies, only the high, medium and low scenarios should be considered. 
Moreover, due to the expected variance in the results of these scenarios, a quorum of runs should be performed to achieve stable outcomes. Alternatively, scenarios generating results close to the overall average could be used.

Compared to real data, the risk of bird strike is overestimated by factor 2.5 to 3.3 in the simulation. No reactions of birds to aircraft are included in the simulation which might be a contributing factor for this overestimate. The offset in recorded bird strikes should be considered when using the simulation environment for further studies. Up to the authors' knowledge, this is the first simulation environment which enables fast-time simulation of bird strike risk at and around airports. As such, it complements HIL applications as introduced by Hale and Stanley (2017) [10]. The simulation environment (Supplementary Materials) is an open-source tool. Therefore, any party interested in applying the model for their purposes are invited to download and modify it with respect to their individual needs.

\section{Conclusions}

This study analysed a simulation environment representing bird and aircraft movements. Here, the focus was to evaluate the robustness and stability of results with regard to recorded bird strikes and near misses. It was found that when considering a sufficient air traffic density, the requirements for reproducibility are met. With regards to this study, this pertains to the scenarios low, medium and high. However, there is an overestimate in number of bird strikes by factor 2.5 to 3.3. Moreover, the simulation environment does not consider birds' reactions to aircraft. By considering these limitations, the simulation environment can serve as a tool for bird strike-related studies. The range of potential applications is wide. For example, long-term effects of additional management measures can be studied and quantified by using avian radar data from prior and after implementing these measures as input for the simulation. In ongoing research, algorithms that delay departing air traffic in case of a predicted bird strike are implemented into the simulation environment. The goal is to evaluate the effects on an airport's runway capacity and safety when applying such a concept. Next to an algorithm considering deterministic bird trajectories, an algorithm including uncertainties in bird movement will be implemented.

Supplementary Materials: The simulation environment is available on github: https://github.com/isabelmetz/ BlueSky-Bird-Strike-Detection/releases/tag/v1.0.0.

Author Contributions: I.C.M. enhanced the simulation tool with modules to represent birds and to determine the occurrence of collisions between birds and aircraft. She developed and implemented the model for bird and flock size distribution. Furthermore, she designed the set-up for the Monte Carlo experiments, ran the simulations and performed the evaluations of the results. T.M. supported the development of the simulation environment. He assisted in the airport flight plan selection and proposed the concept for the calculation of aircrafts' protected zones for the conflict detection algorithm. J.E. contributed to the concept development of an ATC bird alert system as well as the feasibility study. He developed major parts of the simulation tool. He assisted in the software implementation of the runtime-optimized version of the collision detection algorithm. D.K. supported the development of the simulation environment with his air traffic management experience. H.V.G. supported the processing and implementation of the bird movement information. He proposed the Monte Carlo simulations and contributed to their set-up. Furthermore, he provided the relevant ornithological aspects and the related implications of the results. J.K. provided the statistical tools and contributed to the design of the Monte Carlo simulations as well as the statistical evaluation of the results. J.M.H. initiated this study and proposed the conceptual design of the ATC bird alert system. The simulation tool used within this study was initialized and developed by him. All authors contributed critically to the drafts and gave final approval for publication.

Funding: This work was supported by the DLR/DAAD Research Fellowship.

Acknowledgments: The authors would like to express their gratitude to the Royal Netherlands Meteorogical Institute KNMI, the Royal Netherlands Air Force RNLAF and Robin Radar for providing the radar data for this research. We are grateful to Ommo Hüppop for supporting us with hardly accessible literature and helpful recommendations considering the modelling of flock behaviour. We would like to thank the three anonymous reviewers for their thorough reviews and constructive comments.

Conflicts of Interest: The authors declare no conflict of interest. 


\section{References}

1. Bernhardt, G.E.; Blackwell, B.F.; DeVault, T.L.; Kutschbach-Brohl, L. Fatal injuries to birds from collisions with aircraft reveal anti-predator behaviours. Ibis 2010, 152, 830-834. [CrossRef]

2. Dolbeer, R.A.; Weller, J.R.; Anderson, A.L.; Begier, M.J. Wildife Strikes to Civil Aircraft in the United States 1990-2015; Serial Report Number 22; Federal Aviation Administration National Wildlife Strike Database: Washington, DC, USA, November 2015.

3. Thorpe, J. Update to '100 Years of Fatalities and Destroyed Civil Aircraft due to Bird Strikes'. In Proceedings of the 31th Meeting of the World Bird Strike Association, Stavanger, Norway, 25-29 June 2014.

4. Dolbeer, R.A. Increasing Trend of damaging bird strikes with aircraft outside the airport boundary: Implications for mitigation measures. Hum.-Wildl. Interact. 2011, 5, 1235-1248.

5. McKee, J.; Shaw, P.; Dekker, A.; Patrick, K. Approaches to Wildlife Management in Aviation. In Problematic Wildlife. A Cross-Disciplinary Approach; Angelici, F.M., Ed.; Springer: New York, NY, USA, 2016; Chapter 22, pp. $465-488$.

6. Dolbeer, R.A. Height Distribution of Birds Recorded by Collisions with Civil Aircraft. J. Wildl. Manag. 2006, 70, 1345-1350. [CrossRef]

7. Shamoun-Baranes, J.; van Gasteren, H.; Ross-Smith, V. Sharing the aerosphere: Conflicts and potential solutions. In Aeroecology; Springer: New York, NY, USA, 2017; pp. 465-497.

8. ICAO. Wildlife Control and Reduction. In Airport Services Manual, 4th ed.; International Civil Aviation Organization (ICAO): Montreal, QC, Canada, 2012.

9. DeVault, T.L.; Blackwell, B.F.; Seamans, T.W.; Belant, J.L. Identification of off airport interspecific avian hazards to aircraft. J. Wildl. Manag. 2016, 80, 746-752. [CrossRef]

10. Hale, M.R.; Stanley, R. Evaluating the Design and Suitability of the Wildlife Surveillance Concept. In Proceedings of the Integrated Communications Navigation and Surveillance Conference, Herndon, VA, USA, 18-20 April 2017.

11. Dolbeer, R.A. Feathers in the fan. AeroSafety World 2008, 3, 22-26.

12. National Transportation Safety Board NTSB. Loss of Thrust in Both Engines After Encountering a Flock of Birds and Subsequent Ditching on the Hudson River, US Airways Flight 1549, Airbus A320-214, N106US. In Proceedings of the Animation for Aircraft Accident, Weehawken, NJ, USA, 15 January 2009.

13. European Organization for the Safety of Air Navigation. Bird Strike on Final Approach: Guidance for Flight Crews. 2017. Available online: https:/ / www.skybrary.aero/index.php/Bird_Strike_on_Final_Approach: _Guidance_for_Flight_Crews (accessed on 2 February 2018).

14. Metz, I.; Ellerbroek, J.; Mühlhausen, T.; Kügler, D.; Hoekstra, J.M. Simulating the Risk of Bird Strikes. In Proceedings of the 7th SESAR Innovation Days, Serbia, Balkans, 28-30 November 2017.

15. Hoekstra, J.M.; Ellerbroek, J. BlueSky ATC Simulator Project: An Open Data and Open Source Approach. In Proceedings of the 7th International Conference on Research in Air Transportation, Philadelphia, PA, USA, 20-24 June 2016.

16. Metz, I.; Hoekstra, J.M.; Ellerbroek, J.; Kügler, D. Aircraft Performance for Open Air Traffic Simulations. In Proceedings of the AIAA Modeling and Simulation Technologies Conference, Washington, DC, USA, 13-17 June 2016.

17. Kelly, T.; O'Callaghan, M.; Bolger, R. The Avoidance Behaviour Shown by the Rook (Corvus frugilegus) to Commercial Aircraft. In Advances in Vertebrate Pest Management; Pelz, H.J., Crowan, D., Feare, C., Eds.; Filander Verlag: Furth, Germany, 2001; pp. 291-299.

18. Frid, A.; Dill, L. Human-caused disturbance stimuli as a form of predation risk. Conserv. Ecol. 2002, 6, 1-16. [CrossRef]

19. Blackwell, B.F.; Bernhardt, G.E. Efficacy of aircraft landing lights in stimulating avoidance behavior in birds. J. Wildl. Manag. 2004, 68, 725-732. [CrossRef]

20. Beason, R.C.; Nohara, T.J.; Weber, P. Beware the Boojum: Caveats and strengths of avian radar. Hum.-Wildl. Interact. 2013, 7, 16-46.

21. Dokter, A.M.; Baptist, M.J.; Ens, B.J.; Krijgsveld, K.L.; van Loon, E.E. Bird radar validation in the field by time-referencing line-transect surveys. PLoS ONE 2013, 8, e74129. [CrossRef] [PubMed]

22. Gerringer, M.B.; Lima, S.L.; DeVault, T.L. Evaluation of an avian radar system in a midwestern landscape. Wildl. Soc. Bull. 2016, 40, 150-159. [CrossRef] 
23. Eastwood, E. Radar Ornithology; Methuen: London, UK, 1967.

24. Gauthreaux, S.A., Jr. Weather Radar Quantification of Bird Migration. BioScience 2014, 20, 19-21. [CrossRef]

25. Shamoun-Baranes, J.; Alves, J.A.; Bauer, S.; Dokter, A.M.; Húppop, O.; Koistinen, J.; Leijnse, H.; Liechti, F.; van Gasteren, H.; Chapman, J.W. Continental-scale radar monitoring of the aerial movements of animals. Mov. Ecol. 2014, 2, 9. [CrossRef]

26. Nilsson, C.; Dokter, A.M.; Verlinden, L.; Shamoun-Baranes, J.; Schmid, B.; Desmet, P.; Bauer, S.; Chapman, J.; Alves, J.A.; Stepanian, P.M.; et al. Revealing patterns of nocturnal migration using the European weather radar network. Ecography 2018. [CrossRef]

27. Van Gasteren, H.; Holleman, I.; Bouten, W.; van Loon, E.; Shamoun-Baranes, J. Extracting bird migration information from C-band Doppler weather radars. Ibis 2008, 150, 674-686. [CrossRef]

28. Dokter, A.M.; Liechti, F.; Stark, H.; Delobbe, L.; Tabary, P.; Holleman, I. Bird migration flight altitudes studied by a network of operational weather radars. J. R. Soc. Interface 2010, 8. [CrossRef] [PubMed]

29. Larkin, R.P.; Szafoni, R.E. Evidence for widely dispersed birds migrating together at night. Integr. Comp. Biol. 2008, 48, 40-49. [CrossRef] [PubMed]

30. Newton, I. The Migration Ecology of Birds; Academic Press: New York, NY, USA, 2010.

31. Dolbeer, R.A. The History of Wildlife Strikes and Management at Airports. In Wildlife in Airport Environments. Preventing Animal-Aircraft Collisions through Science-Based Management, 1st ed.; DeVault, T.L., Blackwell, B.F., Belant, J.L., Eds.; The John Jopkins University Press: Baltimore, MD, USA, 2013; Chapter 1, pp. 1-6.

32. Lensink, R.; Kwak, R. Vogeltrek over Arnhem in 1983 met een samenvatting over de periode 1981-1983 en methodieken voor het bewerken van telmateriaal. deel I en II. LIMOSA 1985, 59, 152.

33. Dunning, J.B., Jr. CRC Handbook of Avian Body Masses; CRC Press: Boca Raton, FL, USA, 2007.

34. Zuur, B. Nearest neighbour distances in day and night migrating birds. A study using stereophotography. Vogelwarte 1984, 32, 206-218.

35. Hüppop, O.; Dierschke, J.; Exo, K.M.; Fredrich, E.; Hill, R. Bird migration studies and potential collision risk with offshore wind turbines. Ibis 2006, 148, 90-109. [CrossRef]

36. Australian Transport Safety Bureau. Australian Aviation Wildlife Strike Statistics 2006 to 2015; Australian Transport Safety Bureau: Canberra, Australia, 2017.

37. MacKinnon, B. Sharing the Skies. An Aviation Industry Guide to the Management of Wildlife Hazards; Transport Canada: Ottawa, ON, Canada, 2004.

38. Fernández-Juricic, E.; Gaffney, J.; Blackwelll, B.F.; Baumhardt, P. Bird strikes and aircraft fuselage color: A correlational study. Hum.-Wildl. Interact. 2011, 5, 224-234.

39. Air Traffic Control The Netherlands. AD 2.24 EHEH Charts Related to an Aerodrome. AIP The Netherlands, Effective 10 November 2016. Available online: http:/ / www.ais-netherlands.nl/aim/2017-05-11-AIRAC / eAIP/html/index-en-GB.html (accessed on 7 April 2017).

40. Dekker, A.; van Gasteren, H. EURBASE: Military bird strike frequency in Europe. In Proceedings of the International Bird Strike Committee Conference, Athens, Greece, 23-27 May 2005.

41. Ebert, J. Bird Strikes in the German Civil Aviation 2011 to 2015. Vogel und Luftverkehr Online. 2016. Available online: http:/ / www.davvl.de/sites/default/files/2018-06/2016_ebert_vogelschlaege_deutschen_ zivilluftfahrt_11bis15.pdf (accessed on 2 May 2018).

42. Van Gasteren, H.; Both, I.; Shamoun-Baranes, J.; Laloë, J.O.; Bouten, W. GPS logger onderzoek aan Buizerds helpt vogelaanvaringen op militaire vliegvelden te voorkomen. Limosa 2014, 87, 107-116.

(C) 2018 by the authors. Licensee MDPI, Basel, Switzerland. This article is an open access article distributed under the terms and conditions of the Creative Commons Attribution (CC BY) license (http://creativecommons.org/licenses/by/4.0/). 